\title{
Patrick SAVIDAN (dir.), Dictionnaire des inégalités et de la justice sociale
}

Sylvie Mesure

\section{(2) OpenEdition \\ 1 Journals}

Édition électronique

URL : http://journals.openedition.org/ress/5508

DOI : $10.4000 /$ ress. 5508

ISBN : $1663-4446$

ISSN : 1663-4446

Éditeur

Librairie Droz

Édition imprimée

Date de publication : 20 décembre 2019

Pagination : 292-294

ISSN : 0048-8046

Référence électronique

Sylvie Mesure, "Patrick SAVIDAN (dir.), Dictionnaire des inégalités et de la justice sociale », Revue européenne des sciences sociales [En ligne], 57-2 | 2019, mis en ligne le 20 décembre 2019, consulté le 01 décembre 2020. URL : http://journals.openedition.org/ress/5508 ; DOI : https://doi.org/10.4000/ ress.5508

Ce document a été généré automatiquement le 1 décembre 2020.

(c) Librairie Droz 


\title{
Patrick SAVIDAN (dir.), Dictionnaire des inégalités et de la justice sociale
}

\author{
Sylvie Mesure
}

\section{RÉFÉRENCE}

Patrick SAVIDAN (dir.), 2018, Dictionnaire des inégalités et de la justice sociale, Paris, Presses universitaires de France, $1728 \mathrm{p}$.

1 Placé sous l'égide d'Alexis de Tocqueville et de «cette passion de l'égalité » qui anime les sociétés démocratiques, ce riche dictionnaire explore le lien moderne entre inégalités et justice d'un point de vue à la fois descriptif et normatif. Ainsi que le rappelle l'avant-propos donné à son dictionnaire par Patrick Savidan, professeur d'éthique et de philosophie politique, cofondateur en 2002 de l'observatoire des inégalités, tout manquement à l'égalité est en principe, dans de telles sociétés, appréhendé comme la «forme matricielle de l'injustice », en sorte que face à ce qui se présente à elles comme le " problème » des inégalités, la connaissance rigoureuse des faits ne peut aller sans une réflexion sur ce que doit être une véritable justice sociale et sur les moyens permettant au moins de tendre vers elle. C'est ce dont prend acte ce dictionnaire dont l'objectif est de rendre accessible, au-delà du monde académique, un savoir « actualisé, sûr, pluraliste, qui permette d'interroger nos aspirations en matière de justice et les moyens de les réaliser ». L'ambition était élevée et la tâche " écrasante ». Savidan a pourtant su relever le défi. J'avais eu le plaisir de co-diriger avec lui le Dictionnaire des sciences humaines paru en 2006 aux PUF, dont il reprend ici les orientations majeures :

2 - le pluralisme, qui fait droit à la diversité des approches, des courants, des auteurs et des méthodes;

3 - le caractère transdisciplinaire ou pluridisciplinaire de l'entreprise, qui permet d'enrichir la réflexion en faisant dialoguer les différentes sciences humaines entre elles, mais aussi avec la philosophie et, ce qui est original dans ce Dictionnaire des 
inégalités, avec la littérature et les arts - à quoi correspondent des entrées inédites sur le thème traité, telles par exemple que "Art social ", "Bande dessinée et pauvreté ", "Cinéma et question sociale», "Photographie sociale» ou encore «Littérature et injustice ";

- une politique d'auteurs, qui consiste à inviter les spécialistes retenus pour leurs compétences à transmettre leurs connaissances dans un langage rigoureux, mais sans jargon, à restituer la teneur des controverses scientifiques et des débats publics sur un sujet donné, tout en s'engageant personnellement à faire apercevoir aussi bien la complexité de leur objet que la singularité de leur démarche ;

- une volonté d'ouverture et de décentrement : le Dictionnaire des sciences humaines avait mobilisé pas moins de 350 auteurs français et étrangers aux spécialisations unanimement reconnues; Savidan reconduit ici cette démarche en faisant appel à la quasi-totalité des plus grands spécialistes français sur le domaine des inégalités, mais aussi à des auteurs belges, canadiens, américains ou britanniques, avec des signatures parfois encore peu familières en France, mais reconnues internationalement, telles par exemple celles de Anita Silvers sur le handicap, de Sirma Bilge sur l'intersectionnalité ou de Kevin Bales sur les esclavages contemporains. On notera aussi, au compte du décentrement des perspectives, la volonté de se soustraire à un occidentalisme réducteur en donnant leur place à des entrées comme "Justice postcoloniale » ou encore « Justice sociale et sens du juste en Chine».

6 À ces orientations s'ajoutent l'ambition de fournir une compréhension synthétique des objets de recherche évoqués en déployant les multiples niveaux d'appréhensions des questions traitées sans prétendre pour autant à une impossible exhaustivité, ainsi que, surtout, le souci de comprendre notre monde. Un monde profondément travaillé par les inégalités, engagé pour le meilleur et pour le pire dans un processus de globalisation qui, en raison des profondes transformations qu'il impose, et par les effets de problématisation et d'opacification qu'il entraîne, nous conduit sans cesse à remettre l'ouvrage sur le métier et à réélaborer nos schémas intellectuels.

7 Savidan met ici au service de l'entreprise qu'il coordonne son propre investissement sur un terrain qu'il connaît bien, puisqu'il est l'auteur de plusieurs ouvrages sur les inégalités et sur les questions de justice sociale, notamment Repenser l'égalité des chances (Hachette, 2007); Voulons-nous vraiment l'égalité ? (Albin Michel, 2015). C'est là ce qui fait la qualité et l'unité de son ouvrage qui, en ces temps incertains et inquiets, apporte des éléments de réponse à nos multiples interrogations sur le devenir de nos sociétés.

Le Dictionnaire des inégalités et de la justice sociale rassemble les contributions de 170 auteurs et compte 235 articles. Ces derniers se répartissent en trois catégories : la première comprend des articles d'ordre méthodologique et épistémologique et permet d'identifier et d'évaluer les principaux outils mobilisés par les sciences humaines pour comprendre les inégalités ; la deuxième présente les orientations, les familles de pensée qui, aujourd'hui, structurent la réflexion en matière de justice sociale, et il faut souligner ici ce qu'a de véritablement précieux et d'éclairant le croisement des entrées destinées à en aborder les multiples aspects: "Justice climatique», "Justice distributive», «Justice intergénérationnelle», «Justice linguistique », «Justice reproductive », «Justice fiscale », «Justice spatiale », etc., de même que celui par lequel se trouvent déclinés les différents types d'inégalité: "Inégalités culturelles», " Inégalité de salaire et de revenus ", "Inégalités de santé », "Inégalités scolaires », «Inégalités socio-environnementales », «Inégalités urbaines », «Inégalités 
épistémiques", etc. La troisième catégorie d'entrées enfin recense les objets qui mobilisent l'attention des citoyens, des chercheurs et des politiques avec des entrées classiques et incontournables: "L'âge de la retraite, "Les classes moyennes ", "Les classes sociales ", «Les droits sociaux et économiques», «L'État social, «Le salaire minimum ", etc., et d'autres entrées plus novatrices en ce qu'elles font place à des objets que l'on pourrait qualifier d'« émergents»: "Emploi atypique ", "Pauvreté cachée» (missing poor), "Richesse maximale ", ou encore "Plateformes collaboratives ». Le tout est dirigé d'une main de maître par Savidan, dont on peut certes contester certains choix - on est étonné par exemple de voir consacrée une entrée à part entière à Paul Ricoeur ou à Jacques Rancière dans un tel dictionnaire -, mais dont on s'aperçoit que d'autres apportent incontestablement un éclairage inédit sur la problématique traitée, comme par exemple l'extension du domaine des inégalités à la cause animale.

Il est impossible de résumer un dictionnaire. Concluons simplement en disant qu'avec le Dictionnaire des inégalités et de la justice nous disposons d'un outil indispensable à la compréhension du monde contemporain: globalisé, inégalitaire et somme toute injuste.

\section{AUTEURS}

\section{SYLVIE MESURE}

Paris, GEMASS-CNRS, Sorbonne Université 